\title{
Fibrates for the treatment of cholestatic itch (FITCH): study protocol for a randomized controlled trial
}

\author{
Ruth Bolier ${ }^{1 *}$ (D), Elsemieke S. de Vries ${ }^{1}$, Albert Parés ${ }^{2}$, Jeltje Helder ${ }^{1}$, E. Marleen Kemper ${ }^{3}$, Koos Zwinderman ${ }^{4}$, \\ Ronald P. Oude Elferink ${ }^{1}$, Ulrich Beuers ${ }^{1}$ and the Netherlands Association for the Study of the Liver (NASL) \\ Cholestatic Liver Diseases Study Group
}

\begin{abstract}
Background: Pruritus (itch) is a frequent, burdensome and difficult-to-treat symptom in patients with cholestasis. Fibrates are currently under investigation for the treatment of primary biliary cholangitis in patients with a suboptimal response to ursodeoxycholic acid. Moreover, there is empirical evidence for a possible antipruritic effect. We aim to prove this in a randomized controlled trial, including patients with cholestatic liver diseases other than primary biliary cholangitis that are accompanied by pruritus.

Methods: A multicenter investigator-initiated, double-blind, randomized placebo-controlled trial to evaluate the effect of bezafibrate on cholestatic pruritus in 84 adult patients with primary biliary cholangitis or primary/ secondary sclerosing cholangitis. Primary outcome is the proportion of patients with a reduction of itch intensity of $50 \%$ or more (measured on a Visual Analog Scale) after 21 days of treatment with bezafibrate $400 \mathrm{mg}$ qid or placebo. Secondary outcomes include the effect of bezafibrate on a five-dimensional itch score, liver diseasespecific quality of life, serum liver tests and autotaxin activity. Safety will be evaluated through serum parameters for kidney function and rhabdomyolysis as well as precise recording of (serious) adverse events. We provide a schematic overview of the study protocol and describe the methods used to recruit and randomize patients, collect and handle data and perform statistical analyses.
\end{abstract}

Discussion: Given its favorable safety profile and anticholestatic properties, bezafibrate may become the new first-line treatment option for treating cholestatic pruritus.

Trial registration: Netherlands Trial Register, ID: NCT02701166. Registered on 2 March 2016; Netherlands Trial Register, ID: NTR5436. Registered on 3 August 2015.

Keywords: Bezafibrate, Primary biliary cholangitis, Primary sclerosing cholangitis, Secondary sclerosing cholangitis, Pruritus, Itch

\footnotetext{
* Correspondence: a.r.bolier@amc.uva.nl

${ }^{1}$ Tytgat Institute for Liver and Intestinal Research, Department of Gastroenterology and Hepatology, Academic Medical Center, University of

Amsterdam, Meibergdreef 9, 1105 AZ Amsterdam, The Netherlands

Full list of author information is available at the end of the article
} 


\section{Background}

A wide variety of cholestatic conditions are associated with pruritus (itch), including primary biliary cholangitis (PBC, formerly referred to as primary biliary cirrhosis [1]) and primary sclerosing cholangitis (PSC). The pruritus can occur locally or be generalized and is often reported by patients as the most burdensome symptom of their disease. Treatment options are limited as the pathophysiologic mechanism is largely unknown [2]. In our experience, resistant cases report severe sleep deprivation, depression and even suicidal ideations. In some patients, liver transplantation turns out to be the only option left [3].

\section{Fibrates in cholestatic liver diseases}

Ursodeoxycholic acid (UDCA) is the only FDA- and EMAapproved therapy for $\mathrm{PBC}$, improving transplantation-free survival. UDCA-responsive patients generally have similar life expectancy as sex- and age-matched controls [4]. Still, about $40 \%$ of patients do not respond to UDCA. Complementary treatment strategies are thus needed. In recent years, several case reports and pilot studies describe improvement of serum liver function tests upon fibrate treatment in patients with a suboptimal response to UDCA [5-20]. Whether or not fibrates improve transplantationfree survival in addition to UDCA remains to be proven in a currently ongoing phase III trial in $\mathrm{PBC}$ patients (www.clinicaltrials.gov: NCT01654731). Importantly, UDCA does not show benefit for pruritus in $\mathrm{PBC}$ and PSC. According to clinical observations [21], in line with some of the abovementioned reports [14, 17, 22, 23], bezafibrate does potentially have antipruritic properties. Anecdotally, itch complaints occur or recur after stopping the fibrates $[21,22]$.

\section{Bezafibrate as a potential alternative to current guideline-} recommended antipruritic therapies

Rifampicin, the best available evidence-based treatment for the itch of cholestasis [4, 24, 25], has the disadvantage of hepatotoxicity in up to $12 \%$ [26-32] of patients with cholestasis during prolonged treatment. Moreover, rifampicin induces a wide variety of cytochrome P-450 (CYP) enzymes and, therefore, interacts with many different drugs. Fibrates would thus be an attractive alternative treatment for itch as they seem to be safe for long-term administration and seem to provide additional benefits to the course of the disease (at least, for PBC). Other treatment strategies are not as effective as rifampicin (e.g., bile salt sequestrants, naltrexone) or are experimental and much more invasive (e.g., nasobiliary drainage, plasmapheresis, ultraviolet-B phototherapy, liver transplantation) [4].

\section{Aim of this study: to evaluate the antipruritic effect of fibrates}

The itch-relieving effect in the abovementioned studies was not measured as a primary outcome and was thus not systemically objectified by current quantification methods for itch intensity such as the widely used Visual Analog Scale (VAS) (validated in [33], discussed in [34]). Moreover, antipruritic effects were not controlled for while a considerable placebo effect should be taken into account (about 30\% itch reduction was seen upon placebo treatment in one study [35]). Thus, we would like to validate the promising effect of fibrates on itch as a primary outcome in a double-blind, randomized placebo-controlled study.

\section{Proposed molecular antipruritic effect(s) of bezafibrate}

As an agonist of peroxisome proliferator-activated receptors (PPARs), bezafibrate has anti-inflammatory [36-38], anticholestatic [20,39-42] and antifibrotic [43] properties. In the current study we hope to identify its antipruritic mode of action. Our group recently showed that serum levels of lysophosphatidic acid (LPA) and serum autotaxin (ATX) activity, the enzyme forming the bulk of extracellular LPA, correlate with itch intensity in patients with cholestasis [44]. Our working hypothesis is that during cholestasis, increased serum ATX activity causes an increase in LPA-mediated activation of itchspecific sensory nerve endings. Importantly, successful guideline-approved and experimental antipruritic treatments (rifampicin, nasobiliary drainage and albumin dialysis) in patients with cholestasis correlated with a decline in serum ATX activity levels [45]. Thus, secondary objectives of the current study are determination of the effect of bezafibrate on serum ATX activity levels and/or screening for other pruritogens.

\section{Objectives}

The primary objective is to determine the efficacy of bezafibrate in the treatment of moderate to severe cholestatic pruritus. Secondary objectives include efficacy in improvement of fatigue and liver disease-related quality of life and reduction of serum parameters of cholestasis, cholesterol, triglycerides and autotaxin activity. Safety will be evaluated through recording of (serious) adverse events ((S)AEs) and (suspected unexpected) adverse reactions ((SU)SARs) as well as serum parameters for kidney function and rhabdomyolysis. Also, the effect of stopping treatment on all the abovementioned variables will be assessed.

\section{Trial design}

This is a multicenter, investigator-initiated, double-blind, randomized placebo-controlled trial. 


\section{Methods}

Methods are described according to the Standard Protocol Items: Recommendations for Interventional Trials (SPIRIT) 2013 Checklist for interventional trials which is provided in Additional file 2.

\section{Study setting}

A total of nine academic hospitals are participating in this study, eight in the Netherlands and one in Spain (Barcelona). A list of study sites is provided at www.clinicaltrials.gov: NCT02701166.

\section{Inclusion criteria}

Patients of 18 years or older with primary biliary cholangitis (PBC), primary sclerosing cholangitis (PSC) or secondary sclerosing cholangitis (as defined in the clinical practice guidelines of cholestasis from 2009 by the European Association for the Study of the Liver (EASL) [4]) can be included if they report an itch intensity of at least 5 out of $10 \mathrm{~cm}$ on a VAS twice within the week before inclusion with a minimum of 2 and a maximum of 7 days between both itch scores.

\section{Exclusion criteria}

Subjects meeting any of the following criteria will be excluded from participation:

- Primary dermatologic abnormalities associated with pruritus

- Concomitant guideline-recommended as well as experimental antipruritic therapy, e.g., rifampicin, opioid-receptor antagonists (naltrexone, naloxone), serotonin-reuptake inhibitors (sertraline), ondansetron, phenobarbital, propofol, lidocaine, dronabinol, butorphanol, internal or external biliary drainage, extracorporeal albumin dialysis, ultraviolet-B phototherapy

NB. Topical menthol-containing agents are allowed, as well as bile salt sequestrants (colesevelam, cholestyramin) as long as they are taken at least $4 \mathrm{~h}$ before or after intake of the study medication. Incidental use of these agents should be noted by patients in the diary, structural use should be noted on the Case Report Form (CRF)

- Pregnancy, women of childbearing potential not using contraception, breastfeeding

- Cholestasis due to obstruction that requires invasive desobstructive treatment within the time scope of the study (5 weeks) such as endoscopic retrograde cholangiopancreaticography (ERCP) or surgical removal of a tumor compressing the bile duct

- Use of opiates

- Renal insufficiency (creatinine clearance $<60 \mathrm{~mL} / \mathrm{min}$ ) N.B. Concomitant use of UDCA is allowed

\section{Study interventions}

Placebo tablets were developed matching the licenced Bezalip ${ }^{\oplus}$ Retard $400 \mathrm{mg}$ Actavis tablets. The composition of the placebo tablets consists mainly of lactose monohydrate and excipients used were similar to the excipients of the uncoated Bezalip ${ }^{\oplus}$ Retard 400 mg Actavis tablets. Placebo tablets were manufactured under Good Medicinal Practice (GMP) licence.

Additional file 3 provides a flow diagram of the study protocol. Additionally, a schematic overview according to the SPIRIT guidelines is provided in Fig. 1. Participation requires three 30 -min outpatient clinic visits during the course of the study: at day 0 (start of treatment, bezafibrate or placebo), days 21 (end of treatment) and 35 (follow-up 2 weeks after stop of treatment).

\section{Criteria for withdrawal of study subjects}

- Increase in serum transaminases (alanine aminotransferase (AST), alanine aminotransferase (ALT)) above six times the upper limit of normal or an increase of three or more times compared to the start of treatment

\begin{tabular}{|c|c|c|c|c|c|}
\hline \multirow[b]{3}{*}{ TIMEPOINT (days) } & \multirow{3}{*}{$\begin{array}{c}\text { Enrolment } \\
-7 \text { to }-2 \\
\end{array}$} & \multirow{3}{*}{$\begin{array}{c}\text { Allocation } \\
0\end{array}$} & \multicolumn{3}{|c|}{ STUDY PERIOD } \\
\hline & & & \multicolumn{2}{|c|}{ Intervention } & \multirow{2}{*}{$\begin{array}{c}\text { Close out } \\
35\end{array}$} \\
\hline & & & 1 & 21 & \\
\hline \multicolumn{6}{|l|}{ ENROLMENT: } \\
\hline Eligibility screen & $\mathrm{X}$ & & & & \\
\hline Informed consent & $\mathrm{X}$ & & & & \\
\hline Allocation & & $\mathrm{X}$ & & & \\
\hline \multicolumn{6}{|l|}{ INTERVENTIONS: } \\
\hline Bezafibrate or placebo & & & 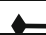 & & \\
\hline \multicolumn{6}{|l|}{ ASSESSMENTS: } \\
\hline VAS \& $5 D$ itch scores & & & $\mathrm{X}$ & $\mathrm{x}$ & $\mathrm{X}$ \\
\hline LSDI2.0 questionnaire & & & $\mathrm{X}$ & $\mathrm{X}$ & $\mathrm{X}$ \\
\hline Serum parameters & & & $\mathrm{X}$ & $\mathrm{X}$ & $\mathrm{x}$ \\
\hline
\end{tabular}

Fig. 1 Standard Protocol Items: Recommendations for Interventional Trials (SPIRIT) figure: schedule of enrollment, interventions and assessments. 5D five-dimensional, LDSI Liver Disease Symptom Index, VAS Visual Analog Scale 
- If serum creatinine is within the normal range at the start of the study, an increase above $133 \mu \mathrm{mol} / \mathrm{L}$ is considered a reason for withdrawal. In case serum creatinine is increased already at start of the study we will allow an increase of $50 \%$ of serum creatinine concentration

\section{Procedures to monitor adherence to treatment}

Tablets remaining after the treatment phase of the intervention will be used to assess adherence to treatment. Moreover, serum cholesterol and triglyceride levels will be tested, reflecting treatment adherence in the bezafibratetreated group.

\section{Outcomes}

\section{Visual Analog Scale (VAS) itch score}

The primary endpoint will be calculated from VAS scores obtained at study visits 1 and 2 (at start and stop of therapy), asking patients to report the maximum itch intensity of the past $24 \mathrm{~h}$. We are using VAS itch scores as it is the most widely used method to assess itch intensity [33]. The scale consists of a $10-\mathrm{cm}$ horizontal line divided into ten equal parts numbered 0 to 10 . The 0 on the left is accompanied by a smiling face and the text "no itch at all," the 10 on the right is accompanied by a sad-looking face and the text "worst itch possible." Mostly to promote treatment fidelity, during the 21-day treatment period, patients will be asked to keep a diary to score their itch intensity (VAS) twice daily (after waking up in the morning and before going to bed in the evening) and make notes such as the use of co-medication, side effects and other information.

\section{Five-dimensional (5D) Pruritus Scale}

The 5D Pruritus Scale [46] is increasingly used in pruritus research, probably more accurately reflecting itch complaints as it evaluates multiple dimensions of itch during the course of the past 2 weeks: in addition to itch intensity (degree, range 1 (not present) to 5 (unbearable)), it assesses duration (range 1 (less than $6 \mathrm{~h}$ a day) to 5 (all day) and direction (range 1 (itch completely resolved) to 5 (getting worse). Disability is rated by the extent to which itch interferes with sleep (range 1 (never affects sleep) to 5 (delays falling asleep and frequently wakes me up at night) and social/leisure activities, homework/errands and professional activities (school/work) (all range 1 (never affects this activity) to 5 (always affects this activity)). Distribution is assessed by marking the affected skin surfaces on a ventral and dorsal body drawing which is an adaption made to the original version of the 5D Pruritus Scale where a list of body parts was listed in order to check affected sites. This way, we aim to calculate the extent of pruritic skin as a percentage of the whole body surface by the "rule of nine," a method widely used to diagnose burn wound surface [47].

\section{Liver Disease Symptom Index (LDSI) version 2.0}

The LDSI2.0 [48] is a short questionnaire used to assess the potential effects of bezafibrate on other liver diseaserelated symptoms. It contains 18 questions regarding complaints of arthritis, pruritus, fatigue, abdominal pain, anorexia, jaundice and psychosocial consequences of the disease including depression and anxiety. Complaints can be rated on a 5-point scale; total score ranges from 18 to 90 , higher scores reflecting worse symptoms.

\section{Biological specimens}

Serum will be collected from each participant at three time points: day 0 , day 21 and day 35. Measurements of serum cholestasis parameters, including bilirubin, alkaline phosphatase (AP), gamma glutamyltransferase $(\gamma \mathrm{GT}), \mathrm{ALT}, \mathrm{AST}$, as well as albumin, lactate dehydrogenase (LDH), creatinine kinase (CK), low- and highdensity lipoprotein (LDL, HDL) and total cholesterol, will be measured at the sites' diagnostic laboratories. Furthermore, sample aliquots will be collected and stored at $-80{ }^{\circ} \mathrm{C}$ until the last patient has completed the trial. Samples will be shipped on dry ice to the principal investigator's laboratory.

Autotaxin (ATX) activity will be measured using an enzymatic endpoint assay, in which the amount of choline produced after $60 \mathrm{~min}$ of incubation of the sample with $1 \mu \mathrm{M}$ of the substrate of ATX, lysophosphatidylcholine (LPC), is detected by fluorescence. Bile acid species will be determined using high-performance liquid chromatography (HPLC).

\section{Sample size calculation}

We will compare the proportion of patients from both treatment arms who show a clinically relevant response to treatment which is defined as a reduction in itch intensity of at least $50 \%$. Based on former placebocontrolled trials for cholestatic itch, selecting data only from patients with itch scores of 5 or higher at baseline who were treated for at least 2 weeks, we estimated that $18 \%$ of placebo-treated patients will show the defined response [30, 35, 49-51]. Based on empiric observations we believe that at least $50 \%$ of bezafibrate-treated patients will respond. Thus, we anticipate a difference in proportions between the bezafibrate and placebo groups of $32 \%$.

Using Fisher's exact test with alpha set at 0.05 we need 38 patients per group for a power of 0.80 for two-sided testing. Taking into account a $10 \%$ possible dropout we will include 84 patients ( 42 per arm) in this study. This power calculation was made after consulting a statistician and using the statistical program nQuery Advisor version 3.0. 


\section{Recruitment}

Patients are recruited from outpatient clinics of participating academic medical centers. After informed consent, based on oral and written information (see Additional file 1) provided by study physician or nurse, patients are enrolled at day 0 if the itch intensity is at least 5 out of 10 on a VAS, and all other inclusion and exclusion criteria are met. For all patients who drop out before day 21, we will include a replacement, whereas patients dropping out during the follow-up phase will not be substituted (see also Additional file 3).

The first participant was enrolled in April 2016. Recruitment is expected to be completed in 18 months, and will be enhanced by creating awareness among clinicians through regular presentation of the trial at (inter)departmental meetings. Moreover, we keep close contact with patient organizations to advertise the trial.

\section{Randomization and blinding}

After informed consent, patients will be allocated randomly to either bezafibrate or placebo treatment (ratio 1:1) as per a computer-generated randomization schedule with randomly varying block sizes (maximum block size of 4) by the sites' investigator through a web-based module written in ALEA (https://nl.tenalea.net, copyright NKI AVL, Amsterdam) by the AMC Clinical Research Unit. Stratification takes place for the VAS itch score at day 0 (2 strata: $5 \mathrm{~cm} \leq \mathrm{VAS}<7.5 \mathrm{~cm}$ versus $7.5 \mathrm{~cm} \leq \mathrm{VAS} \leq 10 \mathrm{~cm})$. An automated email with the resulting randomization number will be sent to the trial pharmacy which allocates the patient to one of the two treatment arms and distributes the study medication accordingly to the patients, using identical packaging for placebo and bezafibrate tablets. This way, patients, physicians and outcome assessors stay blinded for treatment allocation. All investigators are aware that the pharmacy keeps the randomization list locked until all patients have completed the study, and that a request for unblinding can only be done in case a serious adverse event (SAE) occurs. Investigators at all sites keep their own subject identification log, in a secure place, and handle all personal data according to the local regulations on personal data protection.

\section{Data collection and management}

Data collection will take place using electronic Case Report Forms (eCRFs, written by the coordinating investigator using web-based OpenClinica software (www.openclinica.com)) for the three visits, with built-in range checks for most variables. An appointed, independent monitor verifies entry of data on a regular basis by site visits according to a detailed monitoring plan.

From the data collection tool, data can be exported directly to the database software (SPSS). The coordinating investigators manage coded data by regular backups on a secure drive. Site agreements have been signed by coordinating and participating centers to assure agreement about access to, and use of, trial data.

\section{Statistical analysis plan}

Statistical analyses will be performed using SPSS (version 22 or above) according to intention-to-treat principle: patients who dropped out before the end of treatment because of lack of treatment efficacy will be included in sensitivity analyses. The nature and extent of any missing data and coding/typing errors will be addressed in a blinded fashion and dealt with accordingly following general principles (e.g., imputation and/or the use of specific statistical models), as will be described in the resulting manuscript.

Statistical tests will be two-tailed. $P$ values $<0.05$ will be considered as statistically significant. Baseline characteristics of the study population in both groups will be depicted in a table, summarizing means \pm standard deviations of continuous variables and frequencies and proportions (\%) for categorical variables. The primary outcome measure will be depicted in a graph. Specific statistical methods to analyze the most important outcome measures are addressed below. Results of these analyses (\% change in each group, $P$ values) will be presented in a table.

\section{Primary outcome}

The effect of bezafibrate on itch will be confirmed by a Fisher's exact test on the difference in the proportion of patients responding to treatment $(\geq 50 \%$ reduction of itch intensity in the VAS score at day 21 compared to day 0 ) between the bezafibrate and the placebo groups.

\section{Secondary outcomes}

In exploratory analyses, we will test:

- The absolute effect of a 3-week treatment as well as a 2-week follow-up on VAS and 5D Pruritus Scale scores (day 0 versus day 21 and day 21 versus day $35)$ as well as the difference of these effects between both groups (unpaired $t$ tests)

- The effect of a 3-week treatment on daily morning and evening VAS scores in both groups separately (repeated measures analysis of variance (ANOVA) days 1-21)

- The difference in time at which patients reach the primary endpoint (50\% reduction in itch intensity) for the first time between both groups (unpaired $t$ test)

- The difference in morning and evening VAS scores within patients at day 1 (irrespective of treatment group, paired $t$ test) 
- The difference in the effect of a 3-week treatment as well as a 2-week follow-up on LDSI2.0 questionnaire total and subdomain scores between both groups (day 0 versus day 21 and day 21 versus day 35, unpaired $t$ test)

- The difference in the effect of a 3-week treatment as well as a 2-week follow-up on serum ATX activity, AST, ALT, ALP, $\gamma$ GT, bilirubin, albumin, creatinine, CK, LDH, glucose, total cholesterol, HDL- and LDLcholesterol and triglycerides between both groups (day 0 versus day 21 and day 21 versus day 35). Depending on the distribution of the data, parametric or nonparametric tests will be chosen, i.e., unpaired $t$ test and Mann-Whitney $U$ test, respectively

- The number of AEs and SAEs during and after treatment will be listed in a table. As few AEs are expected, no statistical analysis will be applicable to compare between groups

\section{Associative analyses}

Univariable and, if applicable, multivariable analysis will be performed to find out if prerandomization itch intensity stratum, gender, age, Body Mass Index (BMI), underlying liver disease, disease stage and/or use of comedication relate to treatment outcome.

\section{Interim analysis}

As we are not studying an intervention for lifethreatening disease, no interim analysis will be performed to be able to keep investigators blinded until all 84 patients have completed the study.

\section{Discussion}

The treatment of cholestatic pruritus is one of the major challenges in the daily practice of hepatologists. This, together with the promising effect of empirical bezafibrate treatment, was reason for the NASL Cholestatic Liver Diseases Study Group in collaboration with the University of Barcelona to initiate this placebo-controlled trial.

Although rifampicin is available [4], there is considerable resistance among clinicians (and patients) to prescribe (or take) rifampicin due to the risk of hepatotoxicity [26-32]. Bezafibrate would be an attractive alternative given its favorable side-effect profile and disease-modifying properties (in PBC). We choose to first show the efficacy of bezafibrate in a placebocontrolled trial before we consider continuing by showing noninferiority to rifampicin which would require many more participants. A 3-week treatment period seemed appropriate to us, as empirical observations found that this was long enough to perceive an antipruritic effect while we consider that this period of time is still ethically acceptable for patients receiving placebo, as most participants have likely suffered from itch for months or years before participation to the trial (due to, in our experience, patients' and/or doctors' delay and prior trial and error of antipruritic therapies). Because of this relatively short treatment duration, participants are requested not to use any rescue medication during the course of the study other than use of topical agents (e.g., menthol cream) and bile sequestrants (e.g., colestyra$\mathrm{min}$ ), of the use of which should be noted in the diary.

It should be noted that the manufacturer of bezafibrate states that it is contraindicated in patients with liver disease. We believe, however, that with about 20 pilot studies [4-20, 22, 23] performed in patients with PBC and PSC, having increased AP levels as an inclusion criteria and all showing stable or decreasing liver transaminase levels during long-term treatment, it is safe to administer bezafibrate for 3 weeks in our target population.

We choose to only include patients with an itch intensity score of $5 \mathrm{~cm}$ or higher on a 10-cm VAS in order to make the primary endpoint, a reduction of itch intensity of $50 \%$ or more, clinically relevant. We believe the VAS score is the best available but still a subjective measure, and the cutoff point of $5 \mathrm{~cm}$ has been chosen somewhat randomly. To prevent bias we cannot reveal this inclusion criterion to patients. This lack of transparency may cause some indignation, however, especially in those who report an itch intensity score of just below $5 \mathrm{~cm}$ and have persistent itch despite having tried all currently available options. Also, patients who experienced relief of their complaints by taking the study medication may be reluctant to stop treatment after 21 days. For these groups of patients we can only advise clinicians to consider prescribing bezafibrate off label, pending the trial's results.

Given the marked placebo effect on pruritus intensity observed in previous clinical trials, as described above, we have made maximum effort to assure blinding of patients, physicians and study personnel involved. The use of web-based randomization module and subsequent distribution of study medication by a central pharmacy (one in The Netherlands and one in Barcelona) directly to the patients minimizes the risks of unblinding. Patients and their physicians may suspect the nature of study medication administered if, after completing the trial, bezafibrate is prescribed off label, depending on the response to that treatment. We believe, however, this will not affect the reliability of the data yet collected or processes of recruitment and data collection by the involved physician.

Although we are capturing itch intensity data using various measures in parallel (VAS, 5D and LDSI2.0 itch scores during the three visits as well as daily morning and evening VAS scores from the diaries), we endeavor to avoid the statistical multiplicity problem by having 
defined a single confirmatory primary endpoint, based on VAS scores during the study visits. All other data will be used in exploratory analyses only, as defined in the protocol. For example, previous studies described diurnal variation of itch intensity $[2,52]$ where most patients reported more intense itch in the late evening and early nighttime. By using diaries, we will also acquire information on how fast a possible effect occurs. In addition, we hope that the use of a diary stimulates compliance and increases accuracy of co-medication usage and reports of side effects.

\section{Trial status}

Recruitment of patients started in March 2016 and is ongoing at the time of submission of this manuscript.

\section{Additional files}

Additional file 1: Informed consent materials: Patient Information Letter and Informed Consent Form. (PDF $1429 \mathrm{~kb}$ )

Additional file 2: SPIRIT Checklist: overview of items recommended by the SPIRIT guidelines, addressed throughout the manuscript. (DOC $125 \mathrm{~kb}$ )

Additional file 3: Flow diagram of the FITCH study protocol. Abbreviations: 5D five-dimensional, LDSI Liver Disease Symptom Index, VAS Visual Analogue Scale. (PDF $49 \mathrm{~kb}$ )

\section{Abbreviations \\ ALT: Alanine aminotransferase; AMC: Academic Medical Center; ANOVA: Analysis of variance; AP: Alkaline phosphatase; AST: Aspartate aminotransferase; ATX: Autotaxin; BMI: Body Mass Index; CK: Creatinine kinase; CYP: Cytochrome P-450; ERCP: Endoscopic retrograde cholangiopancreaticography; GCP: Good Clinical Practice; GMP: Good Medicinal Practice; HDL: High-density lipoprotein; LDH: Lactate dehydrogenase; LDL: Low-density lipoprotein; LDSI: Liver Disease Symptom Index; LPA: Lysophosphatidic acid (lysophosphatidate); MREC: Medical Research Ethics Committee; NSAIDs: Nonsteroidal anti-inflammatory drugs; PBC: Primary biliary cholangitis (formerly primary biliary cirrhosis); PPAR: Peroxisome proliferator-activated receptor; PSC: Primary sclerosing cholangitis; SAE: Serious adverse event; UDCA: Ursodeoxycholic acid; VAS: Visual Analog Scale; GT: Gamma glutamyltransferase}

\section{Acknowledgements \\ We thank the following members of the Cholestatic Liver Diseases Study Group of the Netherlands Association for the Study of the Liver (NASL) for their input during extensive discussions about the study protocol: Henk R van Buuren, Erasmus Medical Center, Rotterdam; Joost P Drenth, Radboud University Medical Center, Nijmegen; Karel I van Erpecum, University Medical Center Utrecht; Bart van Hoek, Leiden University Medical Center; Peter LM Jansen, Academic Hospital Maastricht; Karin M van Nieuwkerk, Free University Medical Center, Amsterdam; J Marleen de Vree, Groningen University Medical Center.}

\section{Funding}

This study is financed by the Dutch Society of Gastroenterology (NVGE, external, grant 2015-09), Dr. Falk Pharma Benelux (external, grant NL-2015-017-R2) and the Academic Medical Center Foundation (internal, grant 2016/0101), awarded to UB and grant PI15/797 from the Instituto de Salud Carlos III (external), partially supported by the European Regional Development Fund, to AP. The funding parties were not involved in the design of the study and will neither be involved in data collection, analysis and/or interpretation, nor in writing or reviewing the manuscript. The primary sponsor of this trial is Prof. Dr. UH Beuers, Academic Medical Center, Amsterdam, The Netherlands, who is not the main funder but is responsible for initiating, managing, registering and obtaining financial resources for this study. The secondary sponsor is Prof. Dr. A
Parés, Hospital Clínic, Barcelona, Spain, who takes responsibility for managing and obtaining financial resources for the study in Barcelona.

\section{Availability of data and materials}

Scientific publication(s) reporting on the outcomes of this study will be accompanied by complete datasets in a format according to the journals instructions. Until then, only the coordinating and principal investigators have access to the complete dataset, as is contracted in site agreements with all participating sites. The most important results and their implications will be communicated to patient organizations through traditional and social media, after the main scientific publication has been disseminated.

\section{Authors' contributions}

RB wrote the study protocol, coordinated implementation of the protocol at participating sites, organized data management, made applications for funding and wrote the current manuscript. ESV coordinated the implementation of the protocol at participating sites, collects and manages data, recruits and randomizes patients. AP provided intellectual guidance in writing the protocol, recruiting and randomizing patients and collecting data. JH recruits and randomized patients and collects data. EMK provided intellectual guidance in writing the protocol and organized blinded study medication. KZ provided support in writing the statistical analysis plan and performing statistical analyses. RPOE provided intellectual guidance in writing the protocol. UB is the initiating principal investigator, provided intellectual guidance in the writing of the protocol, funding applications and current manuscript, and recruits patients. All authors read and approved the final manuscript.

\section{Competing interests}

The authors declare that they have no competing interests.

\section{Consent for publication}

Not applicable.

\section{Ethics approval and consent to participate}

The study protocol (version 2.0 dated 30-07-2015) was approved by the Medical Research and Ethics Committee (MREC) of the Academic Medical Center in Amsterdam, The Netherlands (reference number 2015-153) for all participating Dutch sites (listed in the protocol) as well as by the Clinical Investigation Ethical Committee of the University Hospital Clinic in Barcelona, Spain (reference number 2015/1031). Any substantial changes to the study protocol and other documents will be filed as an amendment with the aforementioned ethics committees. The study will be conducted in accordance with the Declaration of Helsinki and International Conference on Harmonization-Good Clinical Practice (ICH-GCP) and GMP guidelines. Written informed consent will be obtained from all subjects prior to study inclusion and randomization. Given the negligible risks associated with this study, no Data Safety Monitoring Board has been assigned. However, a trained staff member will perform monitoring at all sites to check the integrity of informed consent procedures, drug accountability and data management according to a detailed monitoring plan.

\section{Participant insurance}

Any harm that study participants may suffer due to participation in the trial is covered by insurance.

\section{Authorship eligibility guidelines}

According to the institutions' research codes, investigators are eligible for authorship of manuscripts related to this study if they contributed substantially to at least the conception and design of the trial and/or data acquisition and/or analysis and interpretation of data, drafted and/or critically reviewed the manuscript and approved its final version. We do not intend the use of professional writers.

\section{Publisher's Note}

Springer Nature remains neutral with regard to jurisdictional claims in published maps and institutional affiliations.

\section{Author details}

${ }^{1}$ Tytgat Institute for Liver and Intestinal Research, Department of Gastroenterology and Hepatology, Academic Medical Center, University of 
Amsterdam, Meibergdreef 9, 1105 AZ Amsterdam, The Netherlands. ${ }^{2}$ Liver Unit, Hospital Clínic, IDIBAPS, CIBERehd, Department of Medicine, University of Barcelona, Barcelona, Spain. ${ }^{3}$ Department of pharmacy, Academic Medical Center, Amsterdam, The Netherlands. ${ }^{4}$ Department of Clinical Epidemiology, Biostatistics and Bioinformatics, Academic Medical Center, Amsterdam, The Netherlands.

Received: 6 December 2016 Accepted: 2 May 2017

Published online: 23 May 2017

\section{References}

1. Beuers U, Gershwin ME, Gish RG, Invernizzi P, Jones DE, Lindor K, et al. Changing nomenclature for PBC: from 'cirrhosis' to 'cholangitis'. Hepatology. 2015;62:1620-2

2. Beuers U, Kremer AE, Bolier AR, Oude Elferink RPJ. Pruritus in cholestasis: facts and fiction. Hepatology. 2014;60:399-407.

3. Kuiper EM, Hansen BE, Metselaar HJ, de Man RA, Haagsma EB, van Hoek B, et al. Trends in liver transplantation for primary biliary cirrhosis in the Netherlands 1988-2008. BMC Gastroenterol. 2010;10:144.

4. Beuers U, Boberg K, Chapman R, Chazouillères OIP, Jones D, Lammert F, et al. EASL clinical practice guidelines: management of cholestatic liver diseases. J Hepatology. 2009;51:237-67.

5. Miyaguchi S, Ebinuma $H$, Imaeda $H$, Nitta $Y$, Watanabe $T$, Saito $H$, et al. A novel treatment for refractory primary biliary cirrhosis? Hepatogastroenterology. 2000;47:1518-21.

6. Nakai S, Masaki T, Kurokohchi K, Deguchi A, Nishioka M. Combination therapy of bezafibrate and ursodeoxycholic acid in primary biliary cirrhosis: a preliminary study. Am J Gastroenterol. 2000;95:326-7.

7. Kurihara T, Maeda A, Shigemoto M, Yamashita K, Hashimoto E. Investigation into the efficacy of bezafibrate against primary biliary cirrhosis, with histological references from cases receiving long term monotherapy. Am J Gastroenterol. 2002;97:212-4.

8. Yano K, Kato H, Morita S, Takahara O, Ishibashi H, Furukawa R. Is bezafibrate histologically effective for primary biliary cirrhosis? Am J Gastroenterol. 2002;97:1075-7.

9. Kanda T, Yokosuka O, Imazeki F, Saisho H. Bezafibrate treatment: a new medical approach for PBC patients? J Gastroenterol. 2003;38:573-8.

10. Itakura J, Izumi N, Nishimura Y, Inoue K, Ueda K, Nakanishi H, et al. Prospective randomized crossover trial of combination therapy with bezafibrate and UDCA for primary biliary cirrhosis. Hepatol Res. 2004;29:216-22.

11. Akbar SM, Furukawa S, Nakanishi S, Abe M, Horiike N, Onji M. Therapeutic efficacy of decreased nitrite production by bezafibrate in patients with primary biliary cirrhosis. J Gastroenterol. 2005;40:157-63.

12. Nakamuta M, Enjoji M, Kotoh K, Shimohashi N, Tanabe $Y$. Long-term fibrate treatment for PBC. J Gastroenterol. 2005;40:546-7.

13. Kita R, Takamatsu S, Kimura T, Kokuryu H, Osaki Y, Tomono N. Bezafibrate may attenuate biliary damage associated with chronic liver diseases accompanied by high serum biliary enzyme levels. J Gastroenterol. 2006:41:686-92.

14. Ohmoto K, Yoshioka N, Yamamoto S. Long-term effect of bezafibrate on parameters of hepatic fibrosis in primary biliary cirrhosis. J Gastroenterol. 2006:41:502-3

15. Hazzan R, Tur-Kaspa R. Bezafibrate treatment of primary biliary cirrhosis following incomplete response to ursodeoxycholic acid. J Clin Gastroenterol. 2010;44:371-3.

16. Takeuchi Y, Ikeda F, Fujioka S, Takaki T, Osawa T, Yasunaka T, et al. Additive improvement induced by bezafibrate in patients with primary biliary cirrhosis showing refractory response to ursodeoxycholic acid. J Gastroenterol Hepatol. 2011;26:1395-401.

17. Lens S, Leoz M, Nazal L, Bruguera M, Pares A. Bezafibrate normalizes alkaline phosphatase in primary biliary cirrhosis patients with incomplete response to ursodeoxycholic acid. Liver Int. 2014;34:197-203.

18. Iwasaki S, Akisawa N, Saibara T, Onishi S. Fibrate for treatment of primary biliary cirrhosis. Hepatol Res. 2007;37:S515-7

19. Iwasaki S, Ohira H, Nishiguchi S, Zeniya M, Kaneko S, Onji M, et al. The efficacy of ursodeoxycholic acid and bezafibrate combination therapy for primary biliary cirrhosis: a prospective, multicenter study. Hepatol Res. 2008;38:557-64.

20. Honda A, Ikegami T, Nakamuta M, Miyazaki T, Iwamoto J, Hirayama T, et al. Anticholestatic effects of bezafibrate in patients with primary biliary cirrhosis treated with ursodeoxycholic acid. Hepatology. 2013;57:1931-41.
21. Reig A, Sese P, Pares A. Bezafibrate: a novel and effective alternative for relieving pruritus in patients with primary biliary cirrhosis. Hepatology. 2015;62:508A.

22. Pares A. Advances in treatment of pruritus and osteoporosis. Abstract book EASL Monothematic Conference: Primary Biliary Cirrhosis, Milan, Italy 2014. J Hepatology. 2014;60:54.

23. Iwasaki S, Tsuda K, Ueta H, Aono R, Ono M, Saibara T, et al. Bezafibrate may have a beneficial effect in precirrhotic primary biliary cirrhosis. Hepatol Res. 1999;16:12-8.

24. Tandon P, Rowe BH, Vandermeer B, Bain VG. The efficacy and safety of bile acid binding agents, opioid antagonists, or rifampin in the treatment of cholestasis-associated pruritus. Am J Gastroenterol. 2007;102:1528-36.

25. Siemens W, Xander C, Meerpohl JJ, Buroh S, Antes G, Schwarzer G, Becker G. Pharmacological interventions for pruritus in adult palliative care patients. Cochrane Database Syst Rev. 2016;11:CD008320.

26. Bachs L, Pares A, Elena M, Piera C, Rodes J. Comparison of rifampicin with phenobarbitone for treatment of pruritus in biliary cirrhosis. Lancet. 1989;1:574-6.

27. Bachs L, Pares A, Elena M, Piera C, Rodes J. Effects of long-term rifampicin administration in primary biliary cirrhosis. Gastroenterology. 1992;102:2077-80.

28. Podesta A, Lopez P, Terg R, Villamil F, Flores D, Mastai R, et al. Treatment of pruritus of primary biliary cirrhosis with rifampin. Dig Dis Sci. 1991;36:216-20.

29. Ghent CN, Carruthers SG. Treatment of pruritus in primary biliary cirrhosis with rifampin. Results of a double-blind, crossover, randomized trial. Gastroenterology. 1988;94:488-93.

30. Woolf GM, Reynolds TB. Failure of rifampin to relieve pruritus in chronic liver disease. J Clin Gastroenterol. 1990;12:174-7.

31. Loginov AS, Reshetniak VI, Petrakov AV. The treatment of primary biliary liver cirrhosis with rifampicin. Ter Arkh. 1993;65:57-62.

32. Prince MI, Burt AD, Jones DE. Hepatitis and liver dysfunction with rifampicin therapy for pruritus in primary biliary cirrhosis. Gut. 2002;50:436-9.

33. Phan NQ, Blome C, Fritz F, Gerss J, Reich A, Ebata T, et al. Assessment of pruritus intensity: prospective study on validity and reliability of the visual analogue scale, numerical rating scale and verbal rating scale in 471 patients with chronic pruritus. Acta Derm Venereol. 2012;92:502-7.

34. Stander S, Blome C, Breil B, Bruland P, Darsow U, Dugas M, et al. Assessment of pruritus - current standards and implications for clinical practice: consensus paper of the Action Group Pruritus Parameter of the International Working Group on Pruritus Research (AGP). Hautarzt. 2012;63:521-2. 4-31.

35. Kuiper EM, van Erpecum KJ, Beuers U, Hansen BE, Thio HB, de Man RA, et al. The potent bile acid sequestrant colesevelam is not effective in cholestatic pruritus: results of a double-blind, randomized, placebo-controlled trial. Hepatology. 2010;52:1334-40.

36. Delerive P, Gervois P, Fruchart JC, Staels B. Induction of IkappaBalpha expression as a mechanism contributing to the anti-inflammatory activities of peroxisome proliferator-activated receptor-alpha activators. J Biol Chem. 2000;275:36703-7.

37. Lee JE, Park JH, Jang SJ, Koh HC. Rosiglitazone inhibits chlorpyrifos-induced apoptosis via modulation of the oxidative stress and inflammatory response in SH-SY5Y cells. Toxicol Appl Pharmacol. 2014;278:159-71.

38. Xu Y, Yang $X$, Wang Z, Li M, Ning $Y$, Chen $S$, et al. Estrogen sulfotransferase (SULT1E1) regulates inflammatory response and lipid metabolism of human endothelial cells via PPARgamma. Mol Cell Endocrinol. 2013;369:140-9.

39. Ghonem NS, Ananthanarayanan M, Soroka CJ. PPAR alpha activates human MDR3/ABCB4 transcription and increases rat biliary phosphatidylcholine secretion. Hepatology. 2014;59:1030-42.

40. Kok T, Bloks WW, Wolters H, Havinga R, Jansen PL, Staels B, et al. Peroxisome proliferator-activated receptor alpha (PPARalpha)-mediated regulation of multidrug resistance 2 (Mdr2) expression and function in mice. Biochem J. 2003;369:539-47.

41. Matsumoto T, Miyazaki H, Nakahashi Y, Hirohara J, Seki T, Inoue K, et al. Multidrug resistance 3 is in situ detected in the liver of patients with primary biliary cirrhosis, and induced in human hepatoma cells by bezafibrate. Hepatol Res. 2004;30:125-36.

42. Shoda J, Inada Y, Tsuji A, Kusama H, Ueda T, Ikegami T, et al. Bezafibrate stimulates canalicular localization of NBD-labeled PC in HepG2 cells by PPARalpha-mediated redistribution of ABCB4. J Lipid Res. 2004;45:1813-25.

43. Miyahara T, Schrum L, Rippe R, Xiong S, Yee HFJ, Motomura K, et al. Peroxisome proliferator-activated receptors and hepatic stellate cell activation. J Biol Chem. 2000;275:35715-22.

44. Kremer AE, Martens JJ, Kulik W, Rueff F, Kuiper EM, van Buuren HR, et al. Lysophosphatidic acid is a potential mediator of cholestatic pruritus. Gastroenterology. 2010;139:1008-18. 
45. Kremer AE, van Dijk R, Leckie P, Schaap FG, Kuiper EM, Mettang T, et al. Serum autotaxin is increased in pruritus of cholestasis, but not of other origin, and responds to therapeutic interventions. Hepatology. 2012;56:1391-400.

46. Elman S, Hynan LS, Gabriel V, Mayo MJ. The 5-D itch scale: a new measure of pruritus. Br J Dermatol. 2010;162:587-93.

47. Waslen GD. Management of outpatient burns. Can Fam Physician. 1986;32:805-8.

48. Van der Plas SM, Hansen BE, de Boer JB, Stijnen T, Passchier J, de Man RA, et al. The Liver Disease Symptom Index 2.0; validation of a disease-specific questionnaire. Qual Life Res. 2004;13:1469-81.

49. Mayo MJ, Handem I, Saldana S, Jacobe H, Getachew Y, Rush AJ. Sertraline as a first-line treatment for cholestatic pruritus. Hepatology. 2007;45:666-74.

50. Terg R, Coronel E, Sorda J, Munoz AE, Findor J. Efficacy and safety of oral naltrexone treatment for pruritus of cholestasis, a crossover, double blind, placebo-controlled study. J Hepatol. 2002;37:717-22.

51. Wolfhagen FH, Sternieri E, Hop WC, Vitale G, Bertolotti M, van Buuren HR. Oral naltrexone treatment for cholestatic pruritus: a double-blind, placebocontrolled study. Gastroenterology. 1997:113:1264-9.

52. Bergasa NV. The itch of liver disease. Semin Cutan Med Surg. 2011;30:93-8.

Submit your next manuscript to BioMed Central and we will help you at every step:

- We accept pre-submission inquiries

- Our selector tool helps you to find the most relevant journal

- We provide round the clock customer support

- Convenient online submission

- Thorough peer review

- Inclusion in PubMed and all major indexing services

- Maximum visibility for your research

Submit your manuscript at www.biomedcentral.com/submit
Biomed Central 